\title{
CLINICAL CASE REPORT \\ Ethical professional practice: exploring the issues for health services to rural Aboriginal communities
}

\author{
JL Malone \\ Athabasca University, St. Paul, Alberta, Canada, and Charles Sturt University, New South Wales,
}

Australia

Submitted: 9 August 2011; Revised: 27 November 2011; Published: 27 February 2012

Malone JL

Ethical professional practice: exploring the issues for health services to rural Aboriginal communities Rural and Remote Health 12: 1891. (Online) 2012

Available: http://www.rrh.org.au

A B S T R A C T

Context: This article provides a first person account of the experience of professional ethics for a psychologist who has worked in several Aboriginal communities in Alberta, Canada.

Issues: These small rural communities tend to have few services and health services are typically provided by multidisciplinary health teams. Team members are predominantly community members, creating an embedded service environment that highlights the need for integrity in relationships. As the psychologist travelling to these communities I require sensitivity to cultural considerations, multiple party responsibilities, and community pressure on service delivery.

Lessons learned: In these settings, in consideration of the principle of respect for the dignity of persons, there is enhanced need for non-discrimination, particularly as most community members are vulnerable persons. Also, the context of small community clinics highlights issues of privacy and confidentiality. Responsible caring in these kinds of general practice also raise ongoing questions about competence and the need for daily risk-benefit analysis. Finally, responsibility to society is also an overarching consideration given the conditions of Canadian Aboriginal communities.

Key words: Aboriginal, Canada, community pressure, cultural context, embedded environments, multidisciplinary, professional ethics, psychology. 


\section{Context}

The case scenario for this article is a psychological practice that has taken place in several small rural Aboriginal communities in Alberta, Canada. These include two Metis Settlements, and four First Nation (Cree and Dene) reserves, with small populations (800-6000 people). The Métis, Cree, and Dene peoples of these communities live in dire socioeconomic circumstances. Services in these communities (referred to in this article as local Aboriginal communities), tend to be funded either directly or indirectly by the Canadian government. Psychological services referred to in this article are those that I have provided as a psychologist travelling to these communities and have been offered within a triage model with local community members who are service providers in their community. Typical presenting issues include trauma, addictions, violence, poverty, and the ongoing effects of colonization. In my role as a health service professional, I have provided therapy, consultations, and psycho-education workshops for community members and service providers.

This article is a first person account of a practicing rural psychologist specific to my work in local small, rural, Aboriginal communities. With that in mind, I will provide a brief personal placement. I am a woman who identifies as a member of the cultural majority and lives and works in a rural Alberta, Canada. I am an academic and practising psychologist who has travelled to work in various local Aboriginal communities over the past 15 years. I was raised in this same rural area with cousins of mixed Aboriginal and non-Aboriginal descent. I experienced and witnessed poverty and racism but had little understanding of the cultures of contexts of life in the surrounding Aboriginal communities. I grew to understand how I was shaped by broader contextual factors in my own life and sought to understand these same experiences for my neighbours. As a professional, I wish to acknowledge the need to consider how broader contextual factors interact to impact practice. My journey has included much learning about culture, consultation, and careful collaborative work in order to become aware of, and address, personal beliefs or biases. This work is ethically necessary and ongoing. Considering a case example of providing services in these local small Aboriginal communities, I reflect on my own learning and the impacts of multiple party responsibility, community pressure, and multicultural considerations for ethical practice.

\section{Issues}

\section{Professional ethics for Canadian psychologists}

...the central dilemma for small community psychologists [is] how to practice at a high ethical level according to ethics codes, and how to also be an engaged member of the small community (p5) ${ }^{1}$.

The Canadian Code of Ethics for Psychologists presents a comprehensive model for ethical decision making that requires four principles to be considered and balanced. When the main principles appear to be in conflict, they are to be considered with the priority weighting given even though the Code is clear that ethical conflicts are complex and that there is no firm weighting for principles. To paraphrase the Canadian Psychological Association code, the recommended steps include ${ }^{2,3}$ :

1. Identify who is potentially affected by the decision.

2. Identify the ethically relevant issues and practices that are pertinent to the dilemma.

3. Consider how issues specific to the psychologist (ie biases, stresses or self-interest) might affect the decision made.

4. Design alternative courses of action.

5. Identify the potential risks and benefits that are associated with each course of action.

6. Carefully select a course of action.

7. Take action with a commitment to assume responsibility for the consequences.

8. Evaluate the consequences of the selected action. 
9. Accept responsibility for the action taken and, if necessary, taking steps to correct any negative consequences that the action may have caused.

10. Take whatever appropriate action to prevent future occurrences of the dilemma.

\section{Multiple party responsibilities}

Current codes of ethics for psychology have increasing emphasis on social responsibility. This responsibility extends beyond individual clients to the families, groups, communities, organizations, and societies that might also be impacted by psychological services ${ }^{4}$. Fisher suggested that psychologists not identify who the client is but rather consider their ethical obligations to all relevant parties ${ }^{5}$. This has implications for informed consent, confidentiality, and other professional ethics issues ${ }^{5}$. As a psychologist, I have professional standards which can be tested by third-party payee expectations, the realities of my own business (when in private practice) or employers, and the demands of my work settings.

In this clinical case scenario services were funded by either the federal government department (responsible for public health for Aboriginal people), or through a provincial postsecondary institution. The federally funded services were originally contracts to provide short-term crisis intervention mental health counselling as designated by the community which were negotiated to reflect a larger range of potential services. The provincially funded services were to provide personal counselling to support the mental health of adult post-secondary students studying in community-based campuses. Requests for services in both contexts have run the gamut typical in generalist practice and the lack of specific guidelines have always tested what services can be provided. Who is the client is not a sufficient question here; rather, what are the ethical responsibilities to the client, the families, the community, the agencies serviced, and/or the funding providers who set out the contracts?

Requests for services: There is often a large wait-list for psychological services in these communities which is further taxed by requests from those who may not be covered by the service. There are requests from community members who do not reside within their community but are willing to commute to receive services. Requests may come from community residents who are not band or community members and sometimes do not have Metis or Treaty status, which means they are directly served by those who fund the service. Family members of students (in the provincial program) may request to attend services together with the student. Requests have also come from neighbouring communities that lack their own services, being even more underserved or remote. In my experience, several of these communities tend to work together and share resources Psychological services are triaged by community members who may determine that a referral is appropriate and promise services that I have not approved. When these referrals present a conflict, I face the difficult dilemma inherent in cultural contexts where giving one's word or making promises carries considerable weight. If my reasons to dispute the appropriateness of referrals appear insensitive or bureaucratic I also risk harm to my relationships within the community and with the triaging teams. There is much negotiation and sharing of information and ideas to balance work by and for the community with the requirements of the external funder and my own ethical and practice guidelines.

Appropriate services: Multiple party responsibility concerns may be may be evidenced in service restrictions. Initially, my contracts specified that I provide only one-onone, short-term crisis services. Community norms have tended to support psychological services for couples, families, and groups. Individual services have sometimes been perceived as more appropriate only for chronic or significant personal issues (which are also not appropriately serviced within a short-term model). Only through consistent advocacy together with the community was I able to influence these contracts to reflect the need to provide psychological health services in more community-normative ways. Now services are provided in individual, family, group, and community-based models each carefully planned with the triage team to balance the boundaries of my contract and ethical service provision with community norms and needs. 
Boundary crossings: Boundary crossings are benign overlapping relationships. They are more common in environments where the psychologist lives in close proximity to clients and may move in the same social circles. Boundary crossings are often unavoidable in rural practice and can be considered a normal and healthy part of rural living ${ }^{6,7}$. In boundary crossings, the psychologist is not 'standoff-ish' but continues to perceive all non-office or non-professional relationships as potentially risky ${ }^{6}$. Boundary violations, however, impair judgement and objectivity and have clear potential to be exploitative and harmful to the client, the professional relationship, and the profession of psychology. Discriminating between a boundary crossing and a boundary violation occurs on a case-by-case basis and is dependent on context, such as the cultural background and theoretical orientation of the client and psychologist ${ }^{8,9}$. Understanding the difference between boundary crossings and boundary violations is complex, even when they appear harmless.

Embedded practice can enhance the psychologist's understanding of community needs and community members ${ }^{1}$ but also poses greater risks. I established familiarity through community involvement. There have been increased requests for services following such activities which may imply that such involvement may have lessened suspicion and increased my approachability. This aspect of an embedded environment may have positively influenced the effectiveness of these rural psychological services. A potentially negative influence is born of my ongoing community and professional work. It is common to know much about various families and community agencies and the embedded nature of the community means that I am often privy to information that makes it a challenge to remain objectives in understanding the phenomenological reality of clients $^{8}$. In this clinical case scenario, psychological services are provided as part of multidisciplinary team within these embedded rural service environments. Other team members tend to be community members and represent professions of counselling, nursing, teaching, and social work. It is not uncommon for referrals to include individuals with whom I have had previous relationships, particularly given the small population within the community. School counsellors for whom I have provided clinical supervision, social workers with whom I have collaborated, or family members with whom I have consulted readily refer themselves. I am open about overlapping relationship risks but have been told that self-referrals are often based on trust developed within previous or overlapping relationships. This is salient as historical abuses by 'outsiders' in Aboriginal communities mean that trust is fundamentally therapeutic and more difficult to foster ${ }^{10}$. Rejecting all overlapping relationships could be seen as disrespectful. Rather, my job becomes careful review and community consultation to assess which relationships are boundary crossings and which might be boundary violations. The risk is breaching professional boundaries in juggling overlapping relationships. The Australian Psychological Society notes that boundary management is one of four most commonly cited ethical issues for rural and remote practitioners $^{8}$.

\section{Community pressure}

Community pressure is the impact of a community's unique values and culture on professional services. This is a significant consideration for rural psychologists ${ }^{5,9}$ as ethical action always takes place within a community and impacts more than just the psychologist and client ${ }^{11}$. Community pressure impacts expectations, visibility, and confidentiality. In this case scenario, both isolation and the local culture of the local Aboriginal communities are salient considerations.

Expectations: For competent and successful practice, rural psychologists must be familiar with the unique values and culture of rural areas overall, and specifically the area in which they practice ${ }^{8,9,12,13}$. There may be an assumption that the rural psychologist is in the role of expert and leader in community development and psychologists in rural practice may need to be perceived positively by the communities in which they work ${ }^{1,6,14}$.

Visibility: Psychologists need to secure trust from key members of the rural social network, many of whom may have considerable knowledge about the psychologists' personal life. This lack of anonymity may develop trust but 
also increases pressure and may pose safety concerns and create anxiety for the psychologist ${ }^{1,8,12,15}$. This may also impact clients as personal information is more readily available (and is often offered to psychologists) through common informal-sharing networks or gossip ${ }^{9,12,16}$. These may reflect small community context or Aboriginal cultural or community contextual differences ${ }^{10}$. Community members are likely to be aware of who is using psychological services, support staff may be familiar with clients, and other agencies may openly share information they have about clients $^{8,12,14}$. These kinds of community pressure become more pronounced as the community size decreases, isolation increases, or where there are increased community expectations $^{3,12}$.

Confidentiality: Given the small size of these communities, traumatic situations can be common knowledge and watching the parking lot is enough to know who comes to my office. Community members may expect psychologists to share privileged information without consent and will see this as a protective and not a harmful act ${ }^{1,8,9,17,18}$. Distinctions needed to be made between confidentiality, anonymity, and privacy for clients, as only confidentiality could be justifiable offered to the client in a rural area ${ }^{19}$. When I first began working in these contexts I struggled greatly to deal with community pressure. What has been helpful for me has been slowly and consistently communicating, negotiating, and advocating for mutually beneficial changes. For example, I negotiated (with funding agencies and community) to have private office space and secure locking storage so that I did not have to transport confidential and sensitive information. More importantly, I needed to develop community awareness and trust. I have sought to do this through attending community functions, volunteering at community events, and struggling to learn and use the local languages. This is not always easy as it requires personal time and provides further opportunities for boundary crossings and violations. In a climate of trauma and in consideration of culture, I have found it to be essential. I have learned much about these communities and as I build trust, client and professional relationships are further fostered.

\section{Multicultural considerations}

I am painfully aware of the historical oppression and colonization of Canadian Aboriginal people and the ongoing social racism that can be replicated in our own services as psychologists. Culturally responsive services require a solid understanding of the social forces and context that shape our lives as well as the lives of our clients ${ }^{10}$.

The Aboriginal worldview is often described as one that includes ${ }^{10,18}$ :

- $\quad$ storytelling

- teaching and sharing circles

- participation in ceremonies

- role modelling of values such as non-interference, non-competitiveness, harmony within the group, responsibility for actions, respect for wisdom of others, emotional restraint, and sharing.

It is for this reason that the one-on-one model mentioned earlier failed to be effective as the only mode of service provision. My greatest concern was that it also does little to foster community development and preventative mental health services because it does not incorporate naturally occurring supports or community resources.

Mental health issues: The reality of the Canadian Aboriginal context is one of over-representation in statistics of suicide, drug and alcohol addiction, domestic violence, unemployment, low income levels, and access of mainstream mental health services ${ }^{10,20}$. This is coupled with insufficient mental health services ${ }^{21,22}$. In my role, I often become a contact for professionals that do not work within the community. Most common are probation or parole officers with the judicial system or specialized service providers like addiction treatment centres or psychiatric facilities. I find it difficult to articulate the essential socio-historical context for client needs without appearing patronizing or appearing to expressing undue sympathy for their situation. My goal is advocacy but I also acknowledge that I may be working with other professionals who have never been exposed to the level 
of poverty, trauma, or abuse that the client may present with. For example, in my experience in these communities it is not uncommon for young adults to live in small homes together with more than 15 people. Food may not be collectively shared in these cramped quarters and processed convenience foods are a common way of ensuring one's limited finances are preserved to feed themselves or their own families. In my professional opinion this can be defined as a form of homelessness and the use of coping skills to manage finances far below the poverty line. Someone unfamiliar with the context might perceive the same situation as the client having a home and demonstrating poor health or financial management, rather than survival skills.

Multigenerational trauma: The historical trauma of Aboriginal Peoples affects their health status and social milieu and informs culturally appropriate services ${ }^{23}$. Prior to colonization, Aboriginal peoples had their own rich social, political, economic, and cultural structures and knowledge systems disregarded by Western cultures gave almost exclusive privilege to their own knowledge systems ${ }^{10}$. Most of these, including those for mental health, were gradually eroded, disregarded, and replaced by Western cultures ${ }^{\mathbf{1 0}}$, resulting in lost social and kinship structures, and marginalization ${ }^{\mathbf{1 0} 24}$. This is perpetuated when services, such as my own, are more closely dictated in their scope by funding agency than by the community itself which best knows and understands its needs. This, however, returns to the issues of multiple party responsibilities in the provision of ethical psychological services.

In these communities there have been multiple suicides in recent years, often involving youth. Often, family dysfunction, substance abuse, and criminal charges are implicated. Culturally, the entire community traditionally mourns each death. Under such circumstances, fatigue and complicated bereavement may have begun to negatively impact community resilience and even optimistic community Elders have questioned the ability of the community to stem such losses.
As professionals we need to take some responsibility for this. It is easy to blame the decisions of the past and to have either apathy or blame in the present. It is difficult to acknowledge what we are each doing and how that is really impacting the lives of Aboriginal Peoples. This extends directly to professionals who work in communities that have had this level of impact from colonization. Typical psychological interventions do not address the social determinants of health in Aboriginal communities that were created through colonization as a result of years of colonization. There needs to be a shift to more efficacious services ${ }^{10,18}$. What we need to do is to assess where we are at and where we have yet to go. My own practice is very different from what it was 15 years ago, and when I look back I am appalled at my own naivety, and how little I understood about the communities, their cultures, and my own role. I trust that means that I will feel the same way in another 15 years. Why should anyone suffer such poverty and discrimination and what is our role? I often ask how I can more appropriately provide services, to work as an ally with the community.

Empirically validated interventions: In this context, impirically validated interventions include:

- $\quad$ providing healing circles ${ }^{25}$

- incorporating concepts of spirituality into holistic service provision ${ }^{25,26}$

- $\quad$ using group interventions ${ }^{27}$

- using 'culture as treatment' (such as a focus on family or lodges), activities that reflect specific cultural values or norms ${ }^{24,26,27}$.

I recommend the resource guides, 'Rooting Mental Health in an Aboriginal World View' ${ }^{10}$ and 'Historical Trauma and Post-Colonial Stress in American Indian Populations ${ }^{\prime 28}$ as they provide a comprehensive reviews of treatment that considers multigenerational trauma among Aboriginal people. In my own practice I have sought to provide more collaborative services together with Elders, local addiction counsellors, and cultural leaders. This is particularly useful in referrals or direct provision of culture-as-treatment options. In one example, mandatory counselling that is court-ordered may be 
negotiated to include sweat lodge ceremonies and Elder visitations rather than merely sessions with me as the psychologist, and I move to more of a case management role in cases where jointly determined to be more appropriate and therapeutic. In other examples, substance use interventions for youth are now held as family talking circles involving naturally occurring community supports, like school counsellors and addiction workers, rather than as one-on-one referrals of the youth to see me for assessment and treatment. The goal is always to promote the strengths within the community, culture, and/or family, rather than the benefit of seeing the psychologist who brings resources external to the clients' normative world.

\section{Lessons learned}

\section{Community pressure}

Rural psychologists must take into account the needs of both client and community requiring a balance between professional and community standards ${ }^{1}$. What if there is community pressure? I have been asked to support community initiatives that have sought to ban known drug dealers from the community. What I have done instead is to assist groups to clarify their reasoning and the historical and cultural ways that they might have to resolve such conflict. This is difficult as I seek to understand and support their own process when I might have personal biases and concerns that run contrary to the group goal.

Psychologists need to gain trust of community members and leaders by being in a professional role at all times within the community $^{1,8}$. Cultural dimensions, like expectations of community involvement, can intensify ethical issues ${ }^{29}$. The suggestion that rural psychologists keep an extremely low profile in the community is likely to contradict culturally relevant practice ${ }^{28}$. Triage models help psychologists to deal with demand by having cases prioritised and it allows shared decision-making on handling caseloads.

\section{Responsible caring}

Rural psychologists experience the double-bind of providing valuable services that may not exist without their expertise and compromising the quality of their services because of the need to work outside their area of training ${ }^{21,22}$. What if community members will not access outside resources because that trust is not established? For my part I seek to use social advocacy with outside resources and to facilitate referrals with extra assistance for clients. Often, unfortunately, the resources do not exist. This is most often experienced with mental health crisis. Even when a community member is suicidal or homicidal the police are reticent to transport them to local or urban hospitals that have limited beds and are likely to release these individuals the next day. Psychiatric stabilization is beyond the scope of my outpatient community services but is frequently requested by family members. Although I support them in seeking external resources I also have no answers to their queries about the lack of appropriate services.

Lack of alternative services has been shown to foster or intensify ethical dilemmas for rural psychologists ${ }^{29}$. Rural psychologists may have a moral responsibility to provide some creative and flexible services beyond their scope of training. This may be questionable ethical practice in urban settings but a defensible ethical stance when working with isolated rural clients. Rural psychologists must be intimately aware of their own comfort levels, their strengths and weaknesses, and how far outside their area of expertise they may be practising ${ }^{12,30}$. Those limits need to be articulated to others and clarified through consultation and supervision specific to generalist practice.

\section{Integrity in relationships}

Rural psychologists who follow strict confidentiality guidelines, decline to share confidential information, or challenge the behaviour of other professionals may alienate them from the community and lose valuable referral sources $^{1,4,8}$. This is perhaps the largest struggle that I have had in these communities. Building effective relationships has 
helped and one successful initiative has been the design and delivery of a workshop on professionalism, ethics, and record-keeping that I provide yearly to community agencies. That is another way of starting conversations to better understand our sometimes conflicting needs and responsibilities.

Maintaining confidentiality is a common challenge ${ }^{21}$, particularly in communities where this is a foreign concept. Wihak and Merali's study of remote practitioners found that confidentiality 'posed unfamiliar challenges in the highly interconnected, small communities where they lived and worked' (p176) ${ }^{21}$. There is a need to balance exposure risks for clients and the psychologist's relationships with community partners ${ }^{9,17}$. There is a need to set these boundaries respectfully, to be clear on the roles and responsibilities of each team member, and the overall need to develop effective working relationships.

\section{Responsibility to society}

Psychologists have a greater responsibility towards the welfare of other members of their society ${ }^{2}$ and special responsibility for social justice ${ }^{2,3}$. Psychologists need to respect naturally occurring support systems, avoiding unnecessary disruption to same, and that actions that may change or enforce these systems must involve relevant community members. In my experience, this is a slow, difficult, but ultimately rewarding process.

Social justice is important in the provision of ethical services to rural people and communities. There is a need to advocate for socially appropriate services which can, at times, mean systemic interventions to deliver appropriate services. The rural practitioner may need to be aware of systemic processes in rural communities, the impact of poverty, and how their professional role can impact these social conditions.

\section{Conclusion}

Contextual factors are essential considerations for ethical professional practice. Culture, communities, and peoples represent but a few of these salient considerations as ethical situations are rarely straightforward and vary by practice setting. This article attempted to highlight some of the challenges of putting ethical guidelines into action from the perspective of a generalist rural and northern practice serving local Aboriginal communities. The balance here involved competing challenges such as confidentiality, dual relationships, cultural sensitivity, and limits of professional competency. In applied practice, I must respect clients' rights, while at the same time providing the best possible service.

In this clinical case example there is enhanced need for nondiscrimination as most clients, and the communities-at-large can be considered vulnerable persons. The context of small community clinic issues highlight privacy and confidentiality concerns. Responsible caring in this kind of general practice raises ongoing questions of competence and the need for daily risk-benefit analysis. Integrity in relationships is the key in such embedded environments as is a responsibility to society when serving Canadian Aboriginal Peoples.

\section{References}

1. Schank JA, Skovholt TM. Ethical practice in small communities: challenges and rewards for psychologists. Washington, DC: American Psychological Association, 2006.

2. Canadian Psychological Association. Canadian code of ethics for psychologists, 3rd edn. Ottawa, ON: Canadian Psychological Association, 2000.

3. Truscott D, Crook KH. Ethics for the practice of psychology in Canada. Edmonton, Alberta: University of Alberta Press, 2004.

4. Blackstock C. Rooting mental health in an Aboriginal worldview. The Provincial Centre of Excellence for Child and Youth Mental Health at CHEO. (Online). 2008. Available: http://www.excellencefor childandyouth.ca/sites/default/files/position_aboriginal_world_vi ew.pdf (Accessed 25 November 2011). 
5. Fisher MA. Replacing “who is the client?" with a different ethical question. Professional Psychology: Research and Practice 2009; 40(1): 17.

6. Zur O. Therapeutic boundaries and dual relationships in rural practice: ethical, clinical and standard of care considerations. Journal of Rural Community Psychology 2006; E9: 1-36.

7. Yonge $\mathrm{O}$, Grundy $\mathrm{Q}$. Managing dual role relationships in rural settings. The CAP Monitor 2006; 24: 2-4.

8. Australian Psychological Society. Guidelines for psychological practice in rural and remote settings. Melbourne, VIC: Australian Psychological Society, 2004.

9. Endacott R, Wood A, Judd F, Hulbert C, Thomas B, Grigg M. Impact and management of dual relationships in metropolitan, regional and rural mental health practice. Australian and New Zealand Journal of Psychiatry 2006; 40: 987-994.

10. Roberts LW, Battaglia J, Epstein RS. Frontier ethics: mental health care needs and ethical dilemmas in rural communities. Psychiatric Services 1999; 50: 497-503.

11. Fisher CB, Fried AL, Masty JK. Critical thinking and ethics in psychology. In: RJ Sternberg HL, Roediger, DF Halpern (Eds). Critical Thinking in Psychology. New York, NY, US: Cambridge University Press, 2007; 271-288.

12. Helbok CM. The practice of psychology in rural communities: potential ethical dilemmas. Ethics Behavior 2003; 13: 367-384.

13. Jameson JP, Blank MB. Rural services: defining problems and developing solutions. Clinical Psychology: Science Practice 2007; 14: 283-298

14. Barbopoulos A, Clark JM. Practising psychology in rural settings: issues and guidelines. Canadian Psychology 2003; 44(4): $410-424$
15. Gale JA, Deprez RD. A public health approach to the challenges of rural mental health service integration. In: BH. Stamm (Ed.). Rural behavioral health care: an interdisciplinary guide. Washington, DC: American Psychological Association, 2003; $95-$ 108.

16. Helbok CM, Marinelli RP, Walls RT. National survey of ethical practices across rural and urban communities. Professional Psychology - Research Practice 2006; 37: 36-44.

17. Essinger S. The application of the 2002 American Psychological Association's ethics code and its standards of conduct for the psychologist practicing in a rural community as compared to the psychologist practicing in an urban community (Dissertation). Minneapolis: Capella University, 2006.

18. Poonwassie A, Charter A. An Aboriginal worldview of helping: empowering approaches. Canadian Journal of Counselling 2001; 35(1): 63-73.

19. Charlebois J. Counsellors' professional and personal perspectives on working and living in a remote northern community (Thesis). Nova Scotia: Acadia University, 2006.

20. Walker K. An exploration of the transmission of historical trauma in urban native Americans (Dissertation). San Francisco: Alliant International University, 2006.

21. Kersting K. Professional pioneering on the frontier. Monitor on Psychology 2003; 34(6): 68.

22. Nelson W, Pomerantz A, Howard K, Bushy A. A proposed rural healthcare ethics agenda. Journal of Medical Ethics 2007; 33: 136-139.

23. Struthers R, Lowe J. Nursing in the Native American culture and historical trauma. Issues in Mental Health Nursing 2003; 24: 257272. 
24. Abadian S. From wasteland to homeland: trauma and the renewal of indigenous peoples and their communities (Dissertation). Cambridge: Harvard University Kennedy School of Government, 2000.

25. Romanow R, Marchildon G. Psychological services and the future of health care in Canada. Canadian Psychology 2003; 44(4): 283-295.

26. Pagans LR Jr . Return of the white buffalo: a heuristic study of Native American people transcending cultural genocide (Dissertation). Cincinnati: Union Institute Graduate College, 2001.

27. Cashin J. Trauma and multigenerational trauma caused by genocide and oppression: a comparison of Western and Native American healing methods (Dissertation). Cincinnati: the Union Institute, 2001.
28. Brave Heart MYH, Deschenie T. Resource guide: historical trauma and post-colonial stress in American Indian populations. Tribal College Journal of American Indian Higher Education 2006; 17(3): 24-27.

29. Wihak C, Merali N. Adaptations of professional ethics among counselors living and working in a remote native Canadian community. Journal of Multicultural Counseling and Development 2007; 35: 169-181.

30. Austin W. The ethics of everyday practice: healthcare environments as moral communities. Advances in Nursing Science 2007; 20: 81-88. 